\title{
A numerical investigation of the time distributed-order diffusion model
}

\author{
Xiuling $\mathrm{Hu}^{1}$ \\ F. $\mathrm{Liu}^{2}$ \\ V. $\mathrm{Anh}^{3}$ \\ I. Turner ${ }^{4}$
}

(Received 4 January 2014; revised 13 October 2014)

\begin{abstract}
Distributed-order differential models are more powerful tools to describe complex dynamical systems than the classical and fractionalorder models because of their nonlocal properties. A time distributedorder diffusion model is investigated. By employing some numerical integration techniques, we approximate the distributed-order fractional model with a multi-term fractional model, which is then solved by an implicit numerical method. The stability and convergence of the numerical method is analyzed. Some numerical results are presented to demonstrate the effectiveness of the method and to exhibit the solution behavior of three different diffusion models.
\end{abstract}

http://journal.austms.org.au/ojs/index.php/ANZIAMJ/article/view/7888 gives this article, (c) Austral. Mathematical Soc. 2014. Published November 14, 2014, as part of the Proceedings of the 11th Biennial Engineering Mathematics and Applications Conference. ISSN 1446-8735. (Print two pages per sheet of paper.) Copies of this article must not be made otherwise available on the internet; instead link directly to this URL for this article. 


\section{Contents}

1 Introduction

C465

2 Implicit numerical method

C467

3 Numerical analysis

C469

4 Numerical experiments

C471

5 Conclusions

C474

References

C476

\section{Introduction}

The diffusion equation is a fundamental mathematical model for the evolution of probability densities: $\partial u(x, t) / \partial t=K \partial^{2} u(x, t) / \partial x^{2}$. It describes a transport process resulting from the random motion of a particle $x(t)$ and is characterized by $\left\langle x^{2}(t)\right\rangle \sim \mathrm{Kt}$. In many applications it is generalized by an anomalous diffusion relationship $\left\langle x^{2}(t)\right\rangle \sim t^{\beta}[2,4$, e.g.]. This leads to the time fractional-order kinetic equation $[3,4]$

$$
{ }_{0}^{C} D_{t}^{\beta} u(x, t)=\frac{\partial^{2} u(x, t)}{\partial x^{2}} \quad \text { or } \quad \frac{\partial u(x, t)}{\partial t}={ }_{0} D_{t}^{1-\beta} \frac{\partial^{2} u(x, t)}{\partial x^{2}},
$$

where ${ }_{0}^{C} D_{t}^{\beta}$ and ${ }_{0} D_{t}^{1-\beta}$ denote the Caputo fractional derivative and the Riemann-Liouville fractional derivative, respectively. A fractional-order kinetic equation is classified as:

- a time fractional subdiffusion model for $0<\beta<1$;

- a classical diffusion model for $\beta=1$;

- a time fractional diffusion-wave model for $1<\beta<2$; and 
- a classical wave model for $\beta=2$.

The fractional spatial and temporal kinetic models are more suitable than the standard diffusion model for describing historical data with long-range dependence because of their nonlocal properties and thus are widely used to describe anomalous diffusion and relaxation phenomena. These models were extended to the class of distributed-order models, which provide more useful tools for modelling non-stationary natural signals [5], especially processes which cannot be described by one single power law [6], and diffusion resulting from materials which are subject to a distribution of temperature, electrostatic field strength or magnetic field strength $[1,7]$.

The time distributed-order derivative is [1]

$$
\mathcal{D}_{t}^{P(\beta)} f(x, t)=\int_{\gamma_{1}}^{\gamma_{2}} P(\beta){ }_{0}^{C} \mathcal{D}_{t}^{\beta} f(x, t) d \beta,
$$

for $\beta \in\left[\gamma_{1}, \gamma_{2}\right], \gamma_{1}, \gamma_{2} \in \mathbb{N}$ and $P(\beta)$ a nonnegative weight function. Then, for $\mathrm{m}-1<\beta<\mathrm{m}$ and $\mathrm{m} \in \mathbb{N}$, the general Caputo derivative is

$$
{ }_{0}^{c} \mathcal{D}_{\mathrm{t}}^{\beta} \mathrm{f}(\mathrm{x}, \mathrm{t})=\frac{1}{\Gamma(\mathrm{m}-\beta)} \int_{0}^{\mathrm{t}} \frac{\partial^{\mathrm{m}} \mathrm{f}(\mathrm{x}, \mathrm{s})}{\partial \mathrm{s}^{\mathrm{m}}}(\mathrm{t}-\mathrm{s})^{\mathrm{m}-\beta-1} \mathrm{ds} .
$$

A distributed-order equation was introduced by Caputo in 1969 and solved in 1995 [8]. The time distributed-order kinetic model [1]

$$
\mathcal{D}_{t}^{P(\beta)} f(x, t)=\frac{\partial^{2} u(x, t)}{\partial x^{2}} \quad \text { for } \quad \beta \in(0,2],
$$

is classified as:

- a time distributed-order diffusion model for $\beta \leqslant 1$;

- a time distributed-order wave model for $\beta>1$; and

- a time distributed-order wave-diffusion model for $0<\beta_{1} \leqslant 1<\beta_{2} \leqslant 2$, where $\beta_{1}=\inf \{\beta \mid \beta \in \operatorname{supp} P(\beta)\}$ and $\beta_{2}=\sup \{\beta \mid \beta \in \operatorname{supp} P(\beta)\}$. 
Different forms of distributed-order fractional kinetic models were studied; however, analyses of the underlying numerical methods are relatively limited [1, 9].

We provide a numerical method for solving the initial boundary value problem of the time distributed-order diffusion model

$$
\mathcal{D}_{t}^{P(\beta)} u(x, t)=\frac{\partial^{2} u(x, t)}{\partial x^{2}}+f(x, t),
$$

where $\beta \in(0,1]$ and $(x, t) \in(0, L] \times(0, T]$. The time distributed-order operator $\mathcal{D}_{t}^{P(\beta)}$ defined in (1) uses a nonnegative weight function which satisfies

$$
0 \leqslant P(\beta), \quad P(\beta) \not \equiv 0, \quad 0<\int_{0}^{1} P(\beta) d \beta<\infty .
$$

The boundary and initial conditions of the model are

$$
\begin{aligned}
& u(0, t)=0 \text { and } u(L, t)=0 \text { for } t \in(0, T] \\
& \mathfrak{u}(x, 0)=\phi_{0}(x) \text { for } x \in(0, L] .
\end{aligned}
$$

In Section 2, by applying numerical integration [9], we first approximate the model by a multi-term diffusion model. Then, in Section 3 we solve the multi-term model using a difference method $[10,11]$. Finally, in Section 4 we demonstrate our numerical approximation with some examples.

\section{Implicit numerical method}

First, we discretize the integration interval $[0,1]$ on the grid $0=\xi_{0}<\xi_{1}<$ $\cdots<\xi_{\mathrm{J}}=1$ and set $\Delta \xi_{\mathrm{j}}=\xi_{\mathrm{j}}-\xi_{\mathrm{j}-1}=1 / \mathrm{J}=\sigma$ and $\beta_{\mathrm{j}}=\frac{1}{2}\left(\xi_{\mathrm{j}}+\xi_{\mathrm{j}-1}\right)=$ $(2 j-1) / 2 J$ for $j=1,2, \ldots, J$ and $J \in \mathbb{N}$. Then, using the mid-point quadrature rule [9],

$$
\mathcal{D}_{t}^{P(\beta)} u(x, t)=\sum_{j=1}^{J} P\left(\beta_{j}\right)_{0}^{C} \mathcal{D}_{t}^{\beta_{j}} u(x, t) \Delta \xi_{j}+\mathcal{O}\left(\sigma^{2}\right) .
$$


Thus, the distributed-order diffusion model (2) is transformed into the multiterm diffusion model

$$
\frac{1}{J} \sum_{j=1}^{J} P\left(\beta_{j}\right)_{0}^{C} \mathcal{D}_{t}^{\beta_{j}} u(x, t)=\frac{\partial^{2} u(x, t)}{\partial x^{2}}+f(x, t), \quad(x, t) \in(0, L] \times(0, T] .
$$

Next, we assume that $u(x, t) \in C^{2}([0, L] \times[0, T])$ and define $x_{i}=$ ih for $i=0,1, \ldots, M$ and $t_{k}=k \tau$ for $k=0,1, \ldots, N$, where $h=L / M$ and $\tau=\mathrm{T} / \mathrm{N}$ are the space and time steps, respectively.

Define the grid functions $U_{i}^{k}=u\left(x_{i}, t_{k}\right)$ and $f_{i}^{k}=f\left(x_{i}, t_{k}\right)$. By using the definition (2), Lemma 4.1 by Sun and $\mathrm{Wu}$ [12], and the second-order central difference formula, we obtain the discrete form of model (2) at the point $\left(x_{i}, t_{k+1}\right)$ :

$$
\begin{aligned}
& \frac{1}{J} \sum_{j=1}^{J} \frac{P\left(\beta_{j}\right)}{\mu_{j}}\left[u_{i}^{k+1}-\sum_{l=1}^{k}\left(b_{k-l}^{\beta_{j}}-b_{k-l+1}^{\beta_{j}}\right) u_{i}^{l}-b_{k}^{\beta_{m}} u_{i}^{0}\right] \\
& =\delta_{\chi}^{2} u_{i}^{k+1}+f_{i}^{k+1}+\mathcal{O}\left(\tau^{2-\beta_{j}}+h^{2}+\sigma^{2}\right),
\end{aligned}
$$

where $b_{k}^{\beta_{j}}=(k+1)^{1-\beta_{j}}-k^{1-\beta_{j}}$ and $\mu_{j}=\tau^{\beta_{j}} \Gamma\left(2-\beta_{j}\right)$ [12, for further details]. Let $r_{i}^{k+1}$ be the local truncation error. Then, since $\beta_{j}=(2 j-1) / 2 \mathrm{~J}$ for $1 \leqslant j \leqslant J$ and $1 / J=\sigma$,

$$
1+\frac{1}{2} \sigma=2-\mathrm{J} \sigma+\frac{1}{2} \sigma \leqslant 2-\beta_{j}=2-j \sigma+\frac{1}{2} \sigma \leqslant 2-\sigma+\frac{1}{2} \sigma=2-\frac{1}{2} \sigma .
$$

Then for all $\tau<1$, we derive

$$
\left|r_{i}^{k+1}\right|=O\left(\tau^{2-\beta_{j}}+h^{2}+\sigma^{2}\right) \leqslant C\left(\tau^{1+\frac{\sigma}{2}}+h^{2}+\sigma^{2}\right) .
$$

For $i=1, \ldots, M-1$ and $k=1, \ldots, N-1$, let $u_{i}^{k}$ be the numerical approximation to $U_{i}^{k}$. Then we obtain the implicit numerical scheme

$$
\frac{1}{J} \sum_{j=1}^{J} \frac{P\left(\beta_{j}\right)}{\mu_{j}}\left[u_{i}^{k+1}-\sum_{l=1}^{k}\left(b_{k-l}^{\beta_{j}}-b_{k-l+1}^{\beta_{j}}\right) u_{i}^{l}-b_{k}^{\beta_{j}} u_{i}^{0}\right]=\delta_{x}^{2} u_{i}^{k+1}+f_{i}^{k+1},
$$


with boundary and initial conditions

$$
\begin{aligned}
& u_{0}^{k}=0 \text { and } u_{M}^{k}=0 \text { for } k=1,2, \ldots, N, \\
& u_{i}^{0}=\phi_{0}\left(x_{i}\right) \text { for } i=0,1, \ldots, M .
\end{aligned}
$$

\section{Numerical analysis}

In this section we analyze the stability and convergence of the implicit numerical method (8)-(10).

We rewrite (2) as

$$
\begin{aligned}
& \left(1+2 G h^{-2}\right) u_{i}^{k+1}-G h^{-2} u_{i-1}^{k+1}-G h^{-2} u_{i+1}^{k+1} \\
& =\frac{1}{J} \sum_{j=1}^{J} \frac{P\left(\beta_{j}\right)}{\mu_{j}} G \sum_{l=1}^{k}\left(b_{k-l}^{\beta_{j}}-b_{k-l+1}^{\beta_{j}}\right) u_{i}^{l}+\frac{1}{J} \sum_{j=1}^{J} \frac{P\left(\beta_{j}\right)}{\mu_{j}} G b_{k}^{\beta_{j}} u_{i}^{0}+G f_{i}^{k+1},
\end{aligned}
$$

for $i=1, \ldots, M-1, k=1, \ldots, N-1$ and $G=J / \sum_{j=1}^{J} \frac{P\left(\beta_{j}\right)}{\mu_{j}}$.

Theorem 1. Let $u_{i}^{k}$, for $0 \leqslant i \leqslant M$ and $0 \leqslant k \leqslant N$, be the solution of scheme (11) with boundary and initial conditions (9)-(10). Then

$$
\left\|u^{k+1}\right\|_{\infty} \leqslant\left\|u^{0}\right\|_{\infty}+G\left(b_{k}^{\beta_{j}}\right)^{-1} \max _{1 \leqslant l \leqslant N}\left|f^{l}\right| .
$$


Proof: We give a simple proof of the theorem using mathematical induction. Let $\max _{1 \leqslant i \leqslant M-1}\left|u_{i}^{j}\right|=\left|u_{i_{0}}^{j}\right|$ for $j=0,1,2, \ldots, N$. For $k=0$,

$$
\begin{aligned}
\left\|u^{1}\right\|_{\infty} & \leqslant\left|u_{i_{0}}^{1}\right|\left(1+2 G h^{-2}-G h^{-2}-G h^{-2}\right) \\
& \leqslant\left|\left(1+2 G h^{-2}\right) u_{i_{0}}^{1}-G h^{-2} u_{i_{0}-1}^{1}-G h^{-2} u_{i_{0}+1}^{1}\right| \\
& \leqslant \frac{1}{J} \sum_{j=1}^{J} \frac{P\left(\beta_{j}\right)}{\mu_{j}} G b_{0}^{\beta_{j}}\left|u_{i_{0}}^{0}\right|+G\left|f_{i_{0}}^{1}\right| \\
& \leqslant\left\|u^{0}\right\|_{\infty}+G\left(b_{0}^{\beta_{j}}\right)^{-1} \max _{1 \leqslant l \leqslant N}\left|f^{l}\right| .
\end{aligned}
$$

Suppose that $\left\|u^{j}\right\|_{\infty} \leqslant\left\|u^{0}\right\|_{\infty}+G\left(b_{j-1}^{\beta_{j}}\right)^{-1} \max _{1 \leqslant l \leqslant N}\left|f^{l}\right|$ for $j=1,2, \ldots, k$. Then, for $j=k+1$, by induction and using a similar argument as for $k=0$, the theorem is proved.

Remark 2. Since $\left(1-\beta_{j}\right)(k+1)^{-\beta_{j}}<b_{k}^{\beta_{j}}<\left(1-\beta_{j}\right) k^{-\beta_{j}}$,

$$
G\left(b_{k}^{\beta_{j}}\right)^{-1} \leqslant \frac{J}{\sum_{j=1}^{J} \frac{P\left(\beta_{j}\right)\left(1-\beta_{j}\right)}{(k+1)^{\beta_{j}} \mu_{j}}} \leqslant \frac{J}{\sum_{j=1}^{J} \frac{\left(1-\beta_{j}\right) P\left(\beta_{j}\right)}{T^{\beta_{j}} \Gamma\left(2-\beta_{j}\right)}} \leqslant \frac{1}{\int_{0}^{1} \frac{(1-\beta) P(\beta)}{T^{\beta} \Gamma(2-\beta)} d \beta} \leqslant C .
$$

Hence, Theorem 1 is equivalent to

$$
\left\|u^{k+1}\right\|_{\infty} \leqslant\left\|u^{0}\right\|_{\infty}+C \max _{1 \leqslant l \leqslant N}\left|f^{l}\right|
$$

Theorem 3. Suppose $\mathfrak{u}(x, \mathrm{t}) \in \mathrm{C}_{x, \mathrm{t}}^{3,2}([\mathrm{a}, \mathrm{b}] \times[0, \mathrm{~T}])$ is the smooth solution of the problem (3)-(5) and $\left\{\mathfrak{u}_{i}^{k} \mid 0 \leqslant i \leqslant M, 0 \leqslant k \leqslant N\right\}$ is the solution of the scheme (8)-(10). Let $\boldsymbol{e}_{i}^{k}=\mathfrak{u}\left(\mathrm{x}_{i}, \mathfrak{t}_{\mathrm{k}}\right)-\mathrm{u}_{\mathrm{i}}^{\mathrm{k}}$ for $0 \leqslant j \leqslant M$ and $0 \leqslant \mathrm{k} \leqslant \mathrm{N}$. Then, for $\mathrm{k} \tau \leqslant \mathrm{T}$,

$$
\left\|e^{k+1}\right\|_{\infty} \leqslant C\left(\tau^{1+\sigma}+h^{2}+\sigma^{2}\right) \quad \text { for } \quad k=1, \ldots, N-1 .
$$


Proof: Subtracting (8) from (6) we obtain the error equation

$$
\begin{aligned}
& \left(1+2 G h^{-2}\right) e_{i}^{k+1}-G h^{-2} e_{i-1}^{k+1}-G h^{-2} e_{i+1}^{k+1} \\
& =\frac{1}{J} \sum_{j=1}^{J} \frac{P\left(\beta_{j}\right)}{\mu_{j}} G \sum_{l=1}^{k}\left(b_{k-l}^{\beta_{j}}-b_{k-l+1}^{\beta_{j}}\right) e_{i}^{l}+G r_{i}^{k+1},
\end{aligned}
$$

for $i=1, \ldots, M-1, k=1, \ldots, N-1$ and where $r_{i}^{k}$ satisfies (7). Using a similar argument to that of Theorem 1, we obtain

$$
\left\|e^{k+1}\right\|_{\infty} \leqslant G\left(b_{k}^{\beta_{j}}\right)^{-1} \max _{1 \leqslant l \leqslant N}\left|r^{l}\right| \leqslant C\left(\tau^{1+\frac{\sigma}{2}}+h^{2}+\sigma^{2}\right),
$$

for $k=1,2, \ldots, N-1$, and then the theorem holds.

\section{Numerical experiments}

In this section, we demonstrate the effectiveness of our numerical scheme and exhibit the solution behavior of three different diffusion models obtained for particular choices of the weight function $P(\beta)$ in Equation (1).

Example 4. Consider the distributed-order problem

$$
\int_{0}^{1} \Gamma(3-\beta)_{0}^{C} \mathcal{D}_{\mathrm{t}}^{\beta} \mathrm{u}(x, \mathrm{t}) \mathrm{d} \beta=\frac{\partial^{2} \mathrm{u}(x, \mathrm{t})}{\partial x^{2}}+\mathrm{f}(x, \mathrm{t}),
$$

with $(x, t) \in[0,1] \times[0,1]$,

$$
\begin{array}{ll}
u(0, t)=0, & u(1, t)=0, \quad t \in[0,1] \\
u(x, 0)=0, & x \in[0,1],
\end{array}
$$

and

$$
f(x, t)=2 x^{2}(1-x)^{2}\left(t^{2}-t\right) / \log t-2 t^{2}\left[x^{2}-4 x(1-x)+(1-x)^{2}\right] .
$$


The exact solution is $u(x, t)=t^{2} x^{2}(1-x)^{2}$.

We first approximate the distributed-order diffusion model (13) with the multi-term model

$$
\frac{1}{J} \sum_{j=1}^{J} \Gamma\left(3-\beta_{j}\right)_{0}^{C} \mathcal{D}_{t}^{\beta_{j}} u(x, t)=\frac{\partial^{2} u(x, t)}{\partial x^{2}}+f(x, t) .
$$

Then, we use the method (8)-(10) to compute the numerical solution of equation (16) with the boundary and initial conditions (14)-(15). Figure 1 displays the profiles of the exact and numerical solutions for this problem with $h=1 / 40, \tau=T / 20, \sigma=1 / 10$ and $h=1 / 50, \tau=T / 50$ and $\sigma=1 / 20$. From Figure 1 it is seen that the numerical solutions are in good agreement with the exact solutions. This demonstrates the effectiveness of our numerical method.

Example 5. Consider the classical diffusion, fractional diffusion and time distributed-order diffusion models

$$
\begin{aligned}
& \frac{\partial u(x, t)}{\partial t}=\frac{\partial^{2} u(x, t)}{\partial x^{2}}, \\
& \frac{\partial^{\beta} u(x, t)}{\partial t^{\beta}}=\frac{\partial^{2} u(x, t)}{\partial x^{2}}, \\
& \int_{0}^{1} P(\beta) \frac{\partial^{\beta} u(x, t)}{\partial x^{\beta}} d \beta=\frac{\partial^{2} u(x, t)}{\partial x^{2}} .
\end{aligned}
$$

All three models are computed on the same domain $(x, t) \in(0,1] \times(0,1]$ with the same initial condition

$$
u(x, 0)=x^{2}\left(1-\chi^{2}\right) \quad \text { for } \quad x \in(0,1],
$$

and the same boundary conditions

$$
u(0, t)=0 \text { and } u(1, t)=0 \text { for } t \in(0,1] .
$$

For $h=1 / 50, \tau=1 / 50$ and $\sigma=1 / 10$, Figure 2 exhibits a comparison of the numerical solutions for the three diffusion models. The numerical 


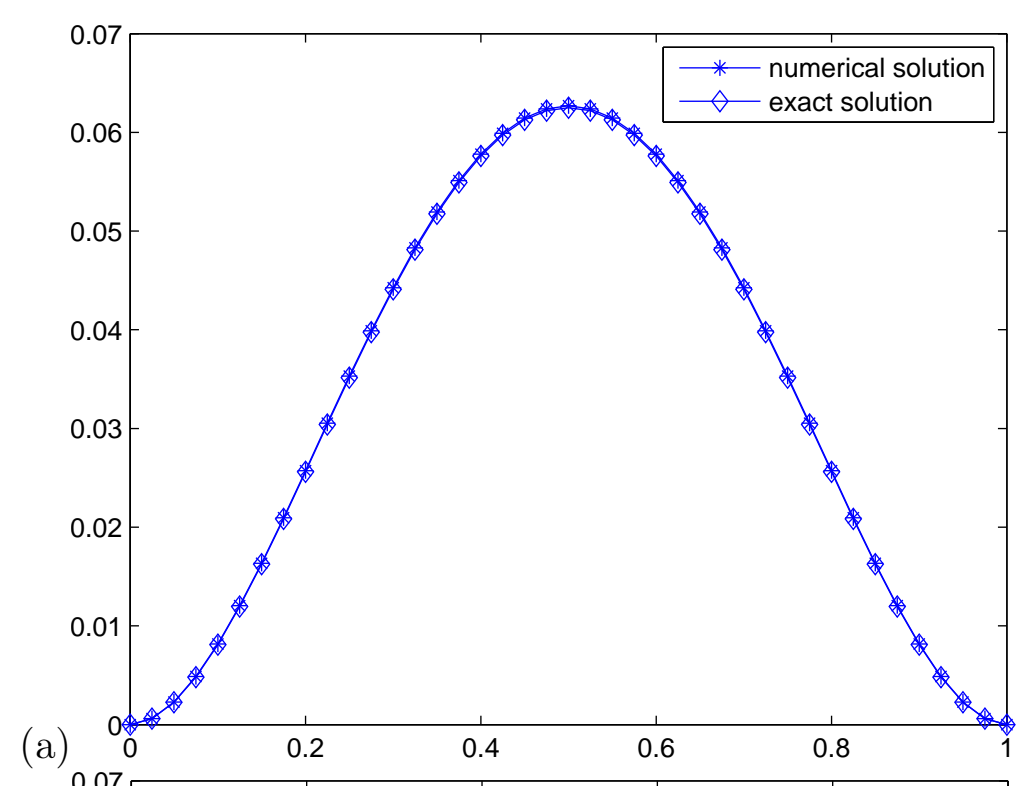

(a)

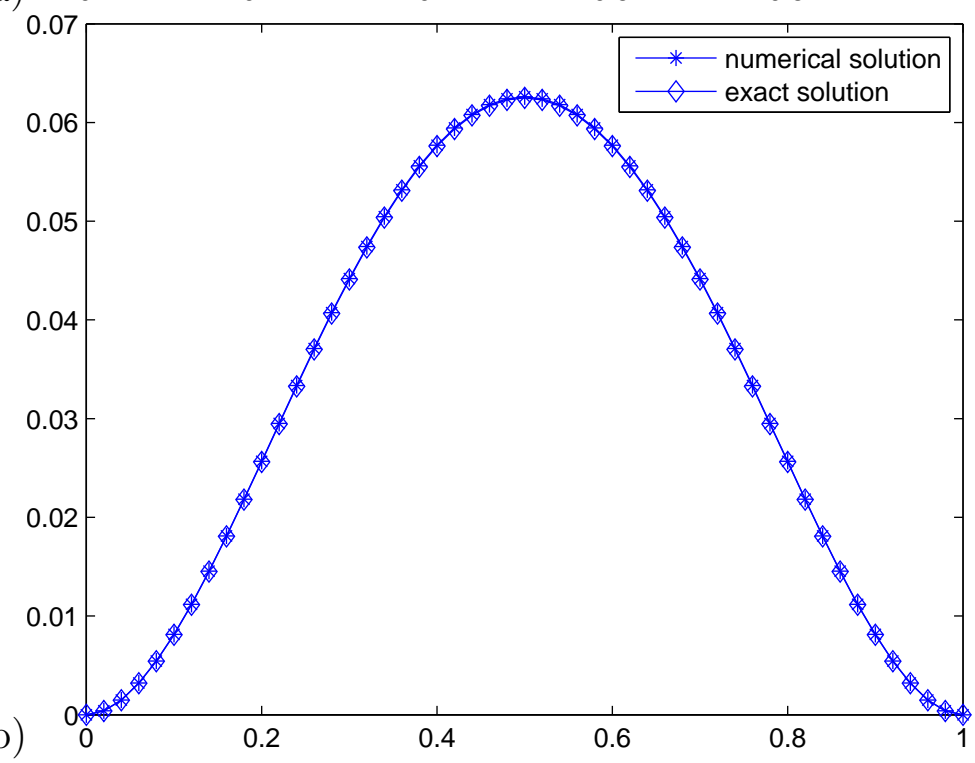

Figure 1: Exact and numerical solutions of $u(x, t)$ from Example 4 over $x \in[0,1]$ for $t=T=1$ and (a) $h=1 / 40, \tau=T / 20, \sigma=1 / 10$; and (b) $h=1 / 50, \tau=T / 50, \sigma=1 / 20$. 
solution of model (17) is computed using the Crank-Nicolson method; the numerical solution of model (18) is computed using method (4.5)-(4.7) of Sun and $\mathrm{Wu}$ [12]; and the numerical solution of model (19) is computed using method (8)-(10). Figure 2 shows the numerical solutions of the classical diffusion model (17), the fractional diffusion model (18) with $\beta=0.5$, and the time distributed-order diffusion model $(19)$ with $P(\beta)=\Gamma(3-\beta)$.

From Figure 2 we see that the diffusion process simulated by the classical model is much faster than those simulated by the other two models. The fractional diffusion model (18) describes sub-diffusion, while the time distributed-order diffusion model (19) depicts retarded random diffusion as described by Sokolov et al. [2] and Chechkin [5]. With different weight functions $P(\beta)$, different diffusion processes, including retarded or accelerated diffusions, are obtained. Thus, the distributed-order diffusion model provides a powerful tool for simulating different complicated dynamical processes with appropriate weight functions of distributed order.

\section{Conclusions}

We investigated an implicit numerical method for the time distributed-order diffusion model. We first approximated the distributed-order diffusion model with a multi-term diffusion model by applying some numerical integration techniques. Then, we provided an implicit numerical method for solving the multi-term model. Stability and convergence of the implicit numerical method were proved. Finally, we gave two numerical examples to exhibit the effectiveness of the method. The first example showed that the numerical simulation is in good agreement with the exact solution. The second example compared the diffusion processes resulting from three mathematical models for different choices of the weight function in the distributed order derivative. 
(a)

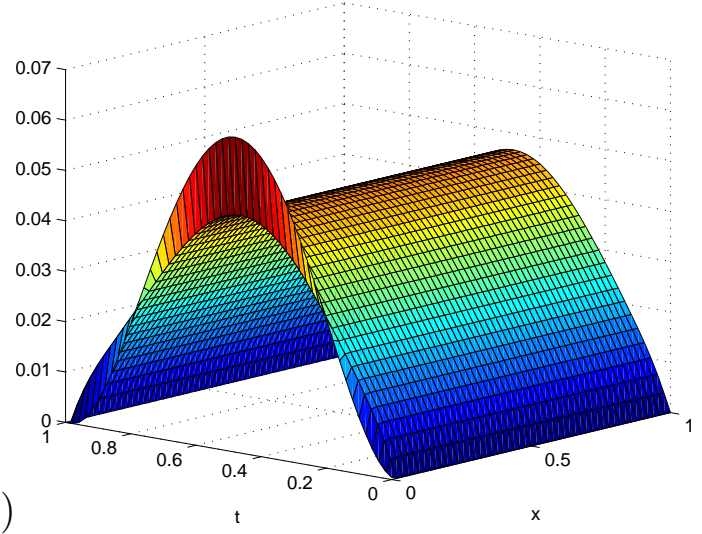

(b)

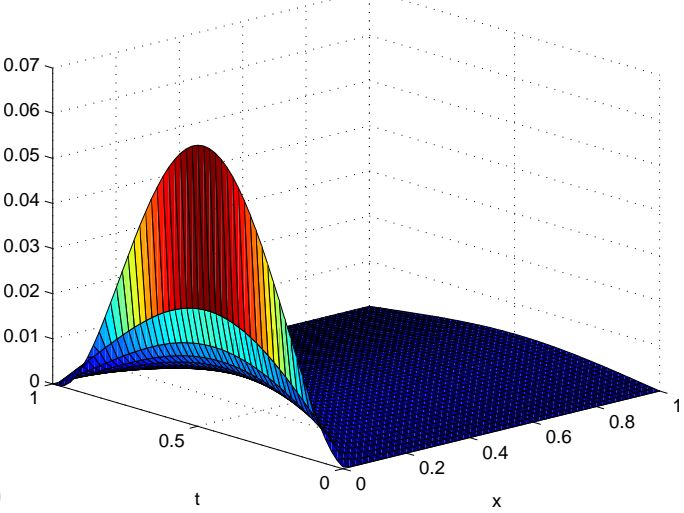

(c)

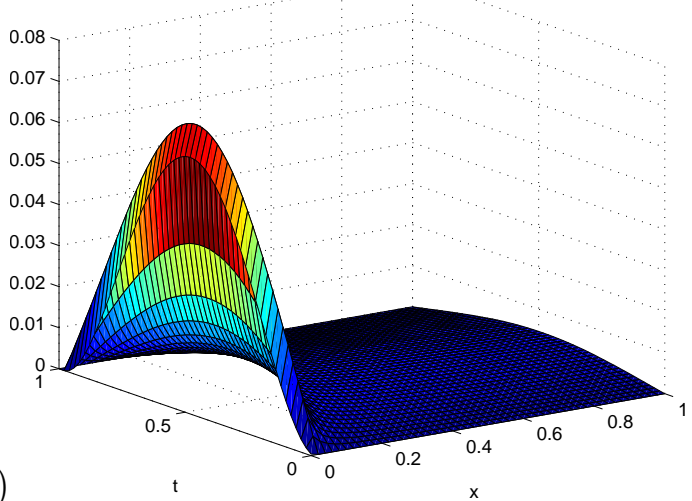

Figure 2: Numerical solutions of $\mathfrak{u}(x, t)$ from Example 5 for (a) classical diffusion; (b) fractional subdiffusion with $\beta=0.5$; and (c) distributed-order diffusion with $P(\beta)=\Gamma(3-\beta)$. 
Acknowledgements The authors thank the referee for the careful reading of the article and many suggestions for its improvement. The first author was supported by the Visiting Scholar Program of Jiangsu Normal University, China. She also thanks QUT for the resources made available to her during her visit. This research is supported by the Australian Research Council grant DP120103770. This work is also partially supported by the National Science Foundation of China under grant 11102179, the Natural Science Foundation of Jiangsu Province under grant BK2012577, and the Natural Science Foundation for Colleges and Universities in Jiangsu Province under grant 12KJB110004.

\section{References}

[1] Z. Jiao, Y. Chen and I. Podlubny, Distributed-Order Dynamic Systems, Springer, 2012. http://www. springer.com/engineering/control/ book/978-1-4471-2851-9. C466, C467

[2] I. M. Sokolov, A. V. Chechkin and J. Klafter, Distributed-order fractional kinetics, Acta Phys. Pol. B, 35:1323-1341, 2004. http: //www .actaphys.uj.edu.pl/_cur/store/vol35/pdf/v35p1323.pdf. C465, C474

[3] F. Liu, P. Zhuang, V. Anh, I. Turner and K. Burrage, Stability and convergence of the difference methods for the space-time fractional advection-diffusion equation, Appl. Math. Comput., 191:12-20, 2007. doi:10.1016/j.amc.2006.08.162. C465

[4] R. Metzler, J. Klafter, The random walk's guide to anomalous diffusion: A fractional dynamics approach, Phys. Rep., 339:1-77, 2000. doi:10.1016/S0370-1573(00)00070-3. C465

[5] A. V. Chechkin, R. Gorenflo and I. M. Sokolov, Retarding subdiffusion and accelerating superdiffusion governed by distributed-order fractional 
diffusion equations, Phys. Rev. E, 66:046129, 2002.

doi:10.1103/PhysRevE.66.046129. C466, C474

[6] M. M. Meerschaert, E. Nane and P. Vellaisamy, Distributed-order fractional diffusions on bounded domains, J. Math. Anal. Appl., 379:216-228, 2011. doi:10.1016/j.jmaa.2010.12.056. C466

[7] C. F. Lorenz and T. T. Hartley, Variable order and distributed order fractional operators, Nonlinear Dynam., 29:57-98, 2002.

http://link. springer.com/article/10.1023/A:1016586905654. C466

[8] M. Caputo, Mean fractional-order-derivatives differential equations and filters. Ann. Univ. Ferrara, 41:73-84, 1995.

http://link. springer.com/article/10.1007\%2FBF02826009. C466

[9] K. Diethelm and N. J. Ford, Numerical analysis for distributed-order differential equations, J. Comp. Appl. Math., 225:96-104, 2009. doi:10.1016/j.cam.2008.07.018. C467

[10] H. Ye, F. Liu, V. Anh and I. Turner, Maximum principle and numerical method for the multi-term time-space Riesz-Caputo fractional differential equations, Appl. Math. Comput., 227:531-540, 2014. doi:10.1016/j.amc.2013.11.015. C467

[11] F. Liu, M. M. Meerschaert, R. J. McGough, P. Zhuang and Q. Liu, Numerical methods for solving the multi-term time-fractional wave-equation, Fract. Calc. Appl. Anal., 16:9-25, 2013. doi:10.2478/s13540-013-0002-2. C467

[12] Z.-Z. Sun and X. Wu, A fully discrete difference scheme for a diffusion-wave system, Appl. Numer. Math., 56:193-209, 2006. doi:10.1016/j.apnum.2005.03.003. C468, C474 


\section{Author addresses}

1. Xiuling $\mathbf{H u}$, School of Mathematics and Statistics, Jiangsu Normal University, Xuzhou, China.

2. F. Liu, School of Mathematical Sciences, Queensland University of Technology, GPO Box 2434, Brisbane, Qld. 4001, Australia. mailto:f.liu@qut.edu.au

3. V. Anh, School of Mathematical Sciences, Queensland University of Technology, GPO Box 2434, Brisbane, Qld. 4001, Australia.

4. I. Turner, School of Mathematical Sciences, Queensland University of Technology, GPO Box 2434, Brisbane, Qld. 4001, Australia. 